Provided for non-commercial research and education use. Not for reproduction, distribution or commercial use.

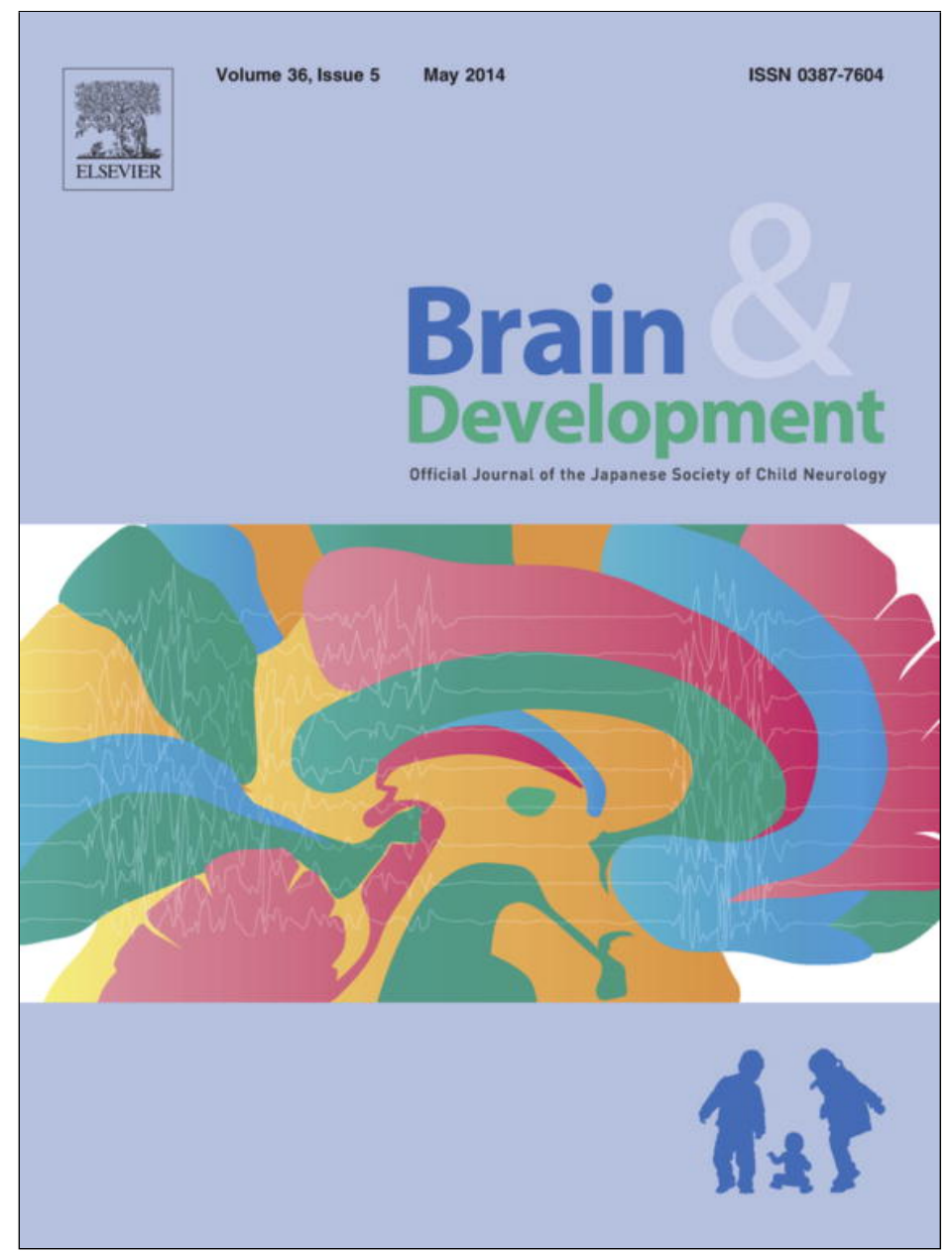

This article appeared in a journal published by Elsevier. The attached copy is furnished to the author for internal non-commercial research and education use, including for instruction at the authors institution and sharing with colleagues.

Other uses, including reproduction and distribution, or selling or licensing copies, or posting to personal, institutional or third party websites are prohibited.

In most cases authors are permitted to post their version of the article (e.g. in Word or Tex form) to their personal website or institutional repository. Authors requiring further information regarding Elsevier's archiving and manuscript policies are encouraged to visit:

http://www.elsevier.com/authorsrights 


\title{
Benign nocturnal alternating hemiplegia of childhood: A new case with unusual findings
}

\author{
Salvatore Mangano ${ }^{\mathrm{a}, *}$, Antonina Fontana ${ }^{\mathrm{a}}$, Chiara Spitaleri ${ }^{\mathrm{a}}$, \\ Giuseppa Renata Mangano ${ }^{\mathrm{b}}$ \\ a Department of Sciences for Health Promotion and Mother and Child Care "G. D'Alessandro", Child Neuropsychiatry Unit, Università di \\ Palermo, Palermo, Italy \\ ${ }^{\mathrm{b}}$ Psychology Department, Università di Palermo, Palermo, Italy
}

Received 12 December 2012; received in revised form 6 June 2013; accepted 7 June 2013

\begin{abstract}
It has been described a neuro developmental disorder labelled "Benign nocturnal alternating hemiplegia of childhood" (BNAHC) characterized by recurrent attacks of nocturnal hemiplegia without progression to neurological or intellectual impairment. We report a female patient who at 11 months revealed a motionless left arm, unusual crying without impairment of consciousness and obvious precipitating factors. The attacks occur during sleep in the early morning with lack of ictal and interictal electroencephalographic abnormalities, progressive neurological deficit, and cognitive impairment. Unlike previous reports of BNAHC our patient come from a family with a history of both migraine, hemiplegic migraine, and sleep disorders. Our study remarks on the typical features described in previous studies and stresses the uncommon aspects that could help to identify the disorder which is likely to have been underestimated. Despite some clinical similarities between BNAHC and familiar hemiplegic migraine and alternating hemiplegia of childhood, the genetic analyses of our patient did not reveal genetic mutations found in both disorders. (c) 2013 The Japanese Society of Child Neurology. Published by Elsevier B.V. All rights reserved.
\end{abstract}

Keywords: Benign nocturnal alternating hemiplegia of childhood; Alternating hemiplegia of childhood; Hemiplegic migraine; Sleep disorders

\section{Introduction}

In the last few years eleven male children have been reported with transient and recurrent attacks of hemiplegia without other paroxysmal neurologic disorders, arising from sleep, and with lack of progression to neurological or intellectual impairment. The attacks typically become less frequent, disappear with aging, and are associated with the normal developmental milestones of children [1-6]. This clinical feature, labelled as "Benign nocturnal alternating hemiplegia of

\footnotetext{
* Corresponding author. Address: U.O. di Neuropsichiatria Infantile, Università di Palermo, Italy. Tel.: +39 091 6555400; fax: +39091 6552048 .

E-mail address: manganos@katamail.com (S. Mangano).
}

childhood" (BNAHC) seems distinct from the classic alternating hemiplegia of childhood (AHC) which is characterized by onset before 18 months, occurrence during wakefulness, ceasing with sleep, associated tonic or dystonic attacks, nystagmus, dyspnoea, and other autonomic phenomena [7]. Furthermore, in the classic form, the attacks are of longer duration and are followed by a progressive cognitive and neurological deterioration over time.

A broad differential diagnosis is necessary when considering this disorder mainly as AHC, hemiplegic migraine, Todd's paralysis, or sleep-related movement disorder.

Here we report a new patient with typical aspects and some unusual manifestations of BNAHC in order to contribute to a better identification of the disorder. 
Furthermore, to investigate whether the BNAHC belongs to the spectrum of AHC and familiar hemiplegic migraine (FHM) we performed the genetic mutational analysis previously described in the latter disorders.

\section{Case report}

Our patient is a 46 months-old girl, the second child born to healthy unrelated parents and has a healthy 6 year old brother. Family history is positive for migraine, hemiplegic migraine (maternal line) and somnambulism (paternal line). She was born at $38 \mathrm{WG}$ following an unremarkable pregnancy by an elective repeat caesarean section. She had normal Apgar score.

At 13 months of age the child was referred to our Child Neuropsychiatric Department at the University of Palermo because at 11 months of age the parents were alerted by the unusual crying of the child who revealed a motionless left arm which lasted $45 \mathrm{~min}$.

On admission, the neurological examination was within normal range. At 14 months and 21 days developmental functioning, assessed by Bayley Scales of Infant Development (BSID-II), showed a mental developmental index (MDI) score within normal limits (97) and a psychomotor developmental index (PDI) score significantly delayed (65).

Repeated interictal waking and sleep EEGs showed bilateral temporal slowing abnormalities. Brain MRI and MR angiography did not detect any structural abnormality. The video-EEG-polysomnography showed a normal sleep pattern, but no attacks could be recorded because of their relative rarity.

Routine blood haematology and chemistry, plasma amino acids profile, lactate and ammonium in the blood, and urinary excretion of organic acids were normal. Mutational analysis of candidate genes of hemiplegic migraine and AHC were negative (ATP1A2, SCN1A, GLUT1, and ATP1A3).

The attack recurred after five, twenty, and sixty days, and nineteen months, and forty-six months, lasting 15, 10, 7, 5 and $4 \mathrm{~min}$, respectively. All episodes involved always the left side, arising from sleep at 6 a.m. without impairment of the consciousness and obvious precipitating factors. All attacks turned off with tremor in the left arm.

At 23 months of age the patient began to experience frequent nocturnal sudden awakening from sleep at 3-6 a.m. associated with abrupt screaming, edginess, and inconsolable crying, lasting a few minutes. During such occurrences, the child held her arm, perhaps to obtain relief from some discomfort, without unilateral or bilateral hypotonia and/or weakness. When the attack remitted she went back to sleep and she did not retain memory of the event but the following day she was irritable and hyperactive. These episodes became gradually rarer and more blurred, but at the same time she developed definite symptoms of oppositional behaviour and hyperactivity often associated with annoying screaming.

At 33 months she had two generalized epileptic seizures requiring an antiepileptic treatment (Valproic acid $30 \mathrm{mg} / \mathrm{kg} /$ day) without further seizure relapse. The postictal EEGs, both performed outside of our Department, displayed some specific abnormalities characterized by diffuse, isolated discharges of low amplitude sharp waves and spikes in the bilateral occipital regions, but dominant on the left side. Whereas, the second EEG displayed bursts of sharp waves on the right frontal regions.

The developmental functioning assessed at 39 months showed a score of 89 for both MDI and PDI of BSID-II. In addition, the clinical evaluation and the "Behavior Rating Scale" of BSID-II revealed negative and irritable emotional tone, poor adaptability, and higher levels of distress.

The last psychological assessment showed a general intellectual functioning in the average (WPPSI III IQ 109). The child showed a change in her behaviour profile characterized by increasing extroversion and gregariousness; she became extremely friendly, with a need to approach other people often inappropriately.

\section{Discussion}

The clinical feature of our patient is characterized by transient nocturnal attacks of hemiplegia without interictal EEG abnormalities, arising from sleep, and recurring during the following years with a progressive decrease in attacks. The occurrence of intellectual function improvement rather than progressive neurological deficit and cognitive impairment supported BNAHC diagnosis rather than the classical AHC.

In this study we emphasized some features that could further contribute to a better identification of the disorder. Indeed, the occurrence of hyperactivity and oppositional behaviour followed by extroversion and gregariousness suggesting difficulties of behavioural and emotional regulation. Furthermore, outside hemiplegia attacks, and during sleep, the child frequently exhibited edginess, and inconsolable crying, which lasted a few minutes, and which were not associated with hypotonia and/or weakness. We think that the behavioural abnormalities could be considered a specific association of this disorder as it has been previously reported in 6 out of 11 patients [2-5]. The sleep events are unusual findings as they have been identified in only one patient [4]. It is unclear if they are a sleeprelated disorder or a mild attack of hemiplegia, but we were impressed by the close negative interference on the behavioural state of the child during the following day.

In addition, our case is a female patient in contrast to the male cases previously investigated, therefore, 
this occurrence is in disagreement with an X-linked trait of inheritance hypothesis [2]. Another unusual clinical characteristic of our patient is the family history of both migraine/FHM and sleep disorders. Although this co-occurrence has never been documented in patients with BNAHC it has been reported that it is not rare in childhood and it has been hypothesized that sleep disorders may be migraine equivalents or precursors suggesting a common neurobiological substrate [8].

The aetiology of BNAHC is unknown but the occurrence of the disorder among siblings and the family history of migraine, or sleep disorder in the previous reported cases seem to suggest the presence of a genetic trait. In addition, some clinical characteristics of the attacks, and their paroxysmal nature induced to hypothesize a relationship with familial hemiplegic migraine (FHM) and AHC. Nevertheless, the genetic mutations, identified to date in both AHC and familial hemiplegic migraine genes (CACNA1A, ATP1A2 and SCN1A), have not been found in BHAHC patients, but to date these genetic analyses were performed on only two out of eleven patients with BHAHC [6].

We expanded the genetic analysis also to GLUT1 as a mutation was found in a patient with AHC [9], and to ATP1A3 because some mutations of this gene were found in $74 \%$ of a large size sample of patients with AHC [10].

Despite some clinical overlaps between BNAHC and FHM/AHC the genetic analyses of our patient did not reveal the genetic mutations found in both FHM and AHC. These results suggest with caution that the BNAHC is a distinct disorder, though the data is still preliminary because only two out of eleven patients were subject to these genetic tests (CACNA1A, ATP1A2, SCN1A) [5].

Our study remarks on the typical features described in previous studies and stresses the uncommon aspects that could help to identify the disorder which is likely to have been underestimated. Further studies need to find additional markers and to elucidate the pathogenetic mechanisms of the BNAHC.

\section{Disclosure section}

Study funding: supported by research funds (ex 60\%) from University of Palermo. The authors report no disclosures.

\section{Acknowledgements}

The authors are grateful to Dr. Federico Zara and Dr. Barbara Garavaglia for the mutational analysis.

We are grateful to Mr. Brinley Thomas for his assistance in checking the translation.

\section{References}

[1] Andermann E, Andermann F, Silver K, Levin S, Arnold D. Benign familial nocturnal alternating hemiplegia of childhood. Neurology 1994;44:1812-4.

[2] Chaves-Vischer V, Picard F, Andermann E, Bernardina B, Andermann F. Benign nocturnal alternating hemiplegia of childhood: six patients and long-term follow-up. Neurology 2001;57:1491-3.

[3] Kavanaugh M, Myers GJ. Benign alternating hemiplegia of childhood: new features and associations. Neurology 2004;62:672.

[4] Sartori S, Vecchi M, Toldo I, Boniver C, Dalla Bernadina B, Laverda AM. Benign nocturnal alternating hemiplegia of childhood: the first clinical report with paroxysmal events home-video recordings. Mov Disord 2008;23:1602-12.

[5] Villéga F, Picard F, Espil-Taris C, Husson M, Michel V, Pedespan JM. Benign nocturnal alternating hemiplegia of childhood: two cases with positive evolution. Brain Dev 2011;33:525-9.

[6] Wagener-Schimmel LJ, Nicolai J. Child neurology: benign nocturnal alternating hemiplegia of childhood. Neurology 2012;79:e161-3.

[7] Bourgeois M, Aicardi J, Goutières F. Alternating hemiplegia of childhood. J Pediatr 1993;122:673-9.

[8] Bruni O, Fabrizi P, Ottaviano S, Cortesi F, Giannotti F, Guidetti V. Prevalence of sleep disorders in childhood and adolescence with headache: a case-control study. Cephalalgia 1997;17:492-8.

[9] Rotstein M, Doran J, Yang H, Ullner PM, Engelstad K, De Vivo DC. GLUT1 deficiency and alternating hemiplegia of childhood. Neurology 2009;73:2042-4.

[10] Heinzen EL, Swoboda KJ, Hitomi Y, Gurrieri F, Nicole S, de Vries B, et al. De novo mutations in ATP1A3 cause alternating hemiplegia of childhood. Nat Genet 2012;44:1030-4. 